\title{
Comparison of consonantal and vocalic cues in selective adaptation
}

\author{
JOHN J. GODFREY \\ Callier Center for Communication Disorders, University of Texas at Dallas, Dallas, Texas 75235
}

\begin{abstract}
The acoustic cues to the phonetic identity of diphthongs normally include both spectral quality and dynamic change. This fact was exploited in a series of selective adaptation experiments examining the possibility of mutual adaptive effects between these two types of acoustic cues. One continuum of syllables varying from $[\varepsilon]$ to $[\varepsilon d]$ and another varying from $[\varepsilon]$ to $[\varepsilon]$ were synthesized; endpoint stimuli of both series used as adaptors caused identification boundaries to be shifted. Cross-series adaptation was also attempted on the

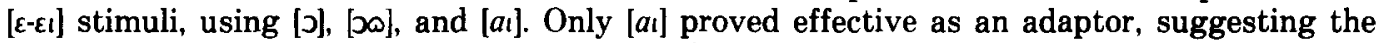
mediation of a rather abstract auditory level of similarity. The results argue strongly against interpretations in terms of feature detectors, but appear compatible with an "auditory contrast" explanation, which might in turn be incorporated within adaptation level theory in the form recently discussed by Restle (1978). The cross-series results further suggest that selective adaptation might be used to quantify the perceptual distance between auditory cues in speech.
\end{abstract}

Until very recently, research with selective adaptation appeared to be making rapid progress in clarifying some of the basic issues in speech perception, at least at the segmental level. There was emerging a picture of a network of neural feature detectors, triggered by specific acoustic properties, capable of rapid and efficient recognition of certain linguistically relevant features of speech sounds. Moreover, the features they could detect seemed to be just the ones underlying the behavior observed in categorical perception, long considered a central issue in speech perception.

Selective adaptation studies generally concentrated on consonantal features-voice onset time (VOT), place of articulation of voiced stops, and manner distinctions. Although it is well known that judgments of vowel identity were liable to change in the presence of contextual differences (Fry, Abramson, Eimas, \& Liberman, 1962; Ladefoged \& Broadbent, 1957), no attempt was made to relate this to the identification changes seen in selective adaptation paradigms until a paper by Morse, Kass, and Turkienicz (1976) suggested a relationship, still in terms of feature detectors. This study at least made it clear that in a selective adaptation paradigm, effects similar to those for consonants are readily obtained with steady-state vowel stimuli from a continuum of formant frequencies. Williams and Sharf (1979) found adaptation of

This research was supported in part by NIH Grant HD 10422-03. Some of the results were presented at 1979 and 1980 meetings of the Acoustical Society of America. The author wishes to thank Kathleen Millay and Ann Syrdal-Lasky for valuable discussions and assistance in preparing the manuscript. Requests for reprints should be addressed to the author at Callier Center for Communication Disorders, 1966 Inwood Road, Dallas, Texas 75235. vowel duration (as a cue to final consonant voicing), and Sawusch and Nusbaum (1979) demonstrated that the vowel adaptation effects of Morse et al. could be achieved with anchoring as well.

Meanwhile, however, the interpretation of these effects in terms of feature detectors has been shown to be problematic. Using different experimental evidence and, to a great extent, different lines of argument, Bryant (1978), Diehl, Elman, and McCusker (1978), and Simon and Studdert-Kennedy (1978) have all concluded that the shifts in consonant identification judgments observed with selective adaptation can be satisfactorily explained without resort to the concept of specialized feature detectors. Simon and Studdert-Kennedy (1978), for example, while agreeing with Sawusch (1977) that two types or levels of effect may be distinguished, argue that neither should be regarded as due to detection of phonetic features. Instead, they suggest the terms "peripheral fatigue" and "central contrast." To the former process they attribute results showing reduced withincategory sensitivity to speech sounds and those identification shifts produced by identical or spectrally similar adaptors, and to the latter process, identification changes associated with more abstract similarities between adaptor and test stimuli. Thus, the demonstration by Bailey $(1973,1975)$ that the actual amount of "spectral overlap" between adaptor and test sounds determines the degree of adaptive shift in identifying place of articulation in stops is taken to reflect (though not exclusively) frequency-specific fatigue. On the other hand, the effects of spectrally displaced adaptors (differing by more than one critical bandwidth from the test stimuli) on the identification of similar consonant series, as in Sawusch (1977, Experiment 2), 
must be due wholly to central contrast. Sawusch confirms this by showing further that such effects show $100 \%$ interaural transfer under dichotic presentation conditions, whereas the effects of spectrally identical adaptors are stronger when presented to the same ear than when presented to the opposite ear.

Still open is the question of whether the effects obtained with vowels and with consonants have the same dual origin. In the recent past parallel questions have been asked in regard to several other perceptual effects: for example, the relative discriminability of vowels and consonants (Pisoni, 1973, Note 1); the right ear advantage in dichotic tasks (Godfrey, 1974; Weiss \& House, 1973); the effect of a suffix on vowels and consonants in precategorical acoustic storage (Darwin \& Baddeley, 1974). Each of these studies demonstrated that a differential effect previously attributed to higher level (i.e., linguistic or phonetic) operations, which presumably treated vowels and consonants separately, was due instead to differences in their respective acoustic stimulus properties. Such findings have been especially helpful in illuminating the role of short-term auditory memory in the speech perception process. If it can be established that adaptation effects with speech sounds, whether vowels or consonants, are likewise dependent only on acoustic properties and not on phonetic status, we would be a step closer to a unified account of these effects, and perhaps of anchoring effects as well.

The experiments described here represent an attempt to bridge the gap between adaptation with consonantal stimuli and adaptation with steady-state vowels and to develop an approach to comparing their relative effectiveness as adaptors. The results suggest that the effects obtained with the two types of adaptors are the same and that, without recourse to the concept of feature detectors, selective adaptation techniques can still yield valuable information about the auditory processing of speech.

It has often been noted that the principal acoustic cues to such consonantal features as place, manner, and voicing of stops are of a transient or rapidly changing character and that they therefore make demands of perception qualitatively different from, say, the steady or slowly varying formant frequencies of vowels. By virtue of both their brevity and their change over time, they appear to place a special burden on the earliest stages of auditory processing, including short-term storage. Surely it was at least in part for this reason that the vast majority of studies on selective adaptation concentrated on sounds distinguished by these cues, for which feature detectors might appear more motivated ecologically (Stevens, 1975). Experiment 1 therefore tested the relative abilities of two stimuli, both containing a brief period of rapid spectral change ([Ed] and $[\varepsilon t]$ ) to adapt responses to a continuum of sounds between them.
Since only one of these stimuli contained a consonant, this experiment constituted a rather strong test of the relevance of the phonetic status of an adaptor.

Experiment 2 again compared two endpoint stimuli as adaptors-an $[\varepsilon]]$ like that of Experiment 1 and a brief steady-state $[\varepsilon]$. Here the presence of brief formant transitions was compared with the absence of any transitions, where both adaptors and the series of sounds between them were phonetically vocalic.

In Experiments 3 and 4, stimulus series varying from $[\varepsilon]$ to $[\varepsilon \iota]$ were again used, and the possibility of "cross-series" adaptation was examined. In Experiment 3, the stimuli were closer to normal syllables in duration $(140 \mathrm{msec})$. The adaptors were [D] and [Do], with first formants identical to, and second formants quite different from, those of the endpoint stimuli of the test series. In Experiment 4, a set of shorter $(81 \mathrm{msec})$ stimuli and adaptors were employed. One adaptor was [Do], identical except in duration to the [Do] adaptor of Experiment 3; the other was $[a t]$, which had neither first nor second formant frequencies in common with any of the test stimuli, but resembled $\left[\varepsilon_{l}\right]$ in the directional pattern of its frequency change over time.

\section{EXPERIMENT 1}

This experiment was designed to compare the effects of consonantal and vocalic adaptors on a single set of test stimuli. At the same time, it constitutes an extreme test of any explanation of adaptation in terms of linguistic feature detectors, since the stimuli of the continuum vary phonemically from the vowel /e/ (as in hay, phonetically $[\varepsilon 1]$ ) to another vowel plus a consonant $/ \varepsilon d /$ (as in head).

\section{Method}

Stimuli. All stimuli were synthesized on a Rockland digital speech synthesizer, Model 4516 (Rabiner, 1968), which is a serial formant-type hardware synthesizer, controlled by a PDP-11/45 computer. For Experiment 1, a continuum of six sounds, varying from $[\varepsilon l]$ to $[\varepsilon d]$, was synthesized. All began with steady-state formant frequencies appropriate for $[\varepsilon]: 500 \mathrm{~Hz}(F 1), 1,700 \mathrm{~Hz}$ $(\mathrm{F} 2)$, and $2,500 \mathrm{~Hz}(\mathrm{~F} 3)$. In stimulus $1, \mathrm{~F} 1$ went from 500 to $400 \mathrm{~Hz}$ and $\mathrm{F} 2$ went from 1,700 to $2,200 \mathrm{~Hz}$; in Stimulus $6, \mathrm{~F} 1$ went from 500 to $200 \mathrm{~Hz}$ and F2 went from 1,700 to $1,800 \mathrm{~Hz}$. Intermediate stimuli had transitions with terminal frequencies at equal logarithmic intervals between these values. In all other respects, Stimuli 1-6 were identical; their total duration was $119 \mathrm{msec}$, with $84 \mathrm{msec}$ of steady state and $35 \mathrm{msec}$ of transitions. All had F3 transitions of $2,500-2,750 \mathrm{~Hz}, \mathrm{~F} 4$ and $\mathrm{F} 5$ fixed at 3,500 and $4,500 \mathrm{~Hz}$, and fundamental frequency $\left(f_{0}\right)$ changing linearly from 111 to $125 \mathrm{~Hz}$. See Table 1 for the acoustic characteristics and Figure 1 for schematic spectrograms of the stimuli.

Subjects. Twelve volunteers with normal speech and hearing served as subjects. All were either students or staff members at the Callier Center for Communication Disorders.

Procedure. Two baseline identification tests were recorded, each containing 10 occurrences of the six stimuli in different random orders, with a 3-sec interstimulus interval (ISI), for a total of 60 unadapted identification responses per test. Two adaptation 
Table 1

Initial (Steady-State) and Final Frequencies (in Hertz) of the First Three Formants of the Stimuli

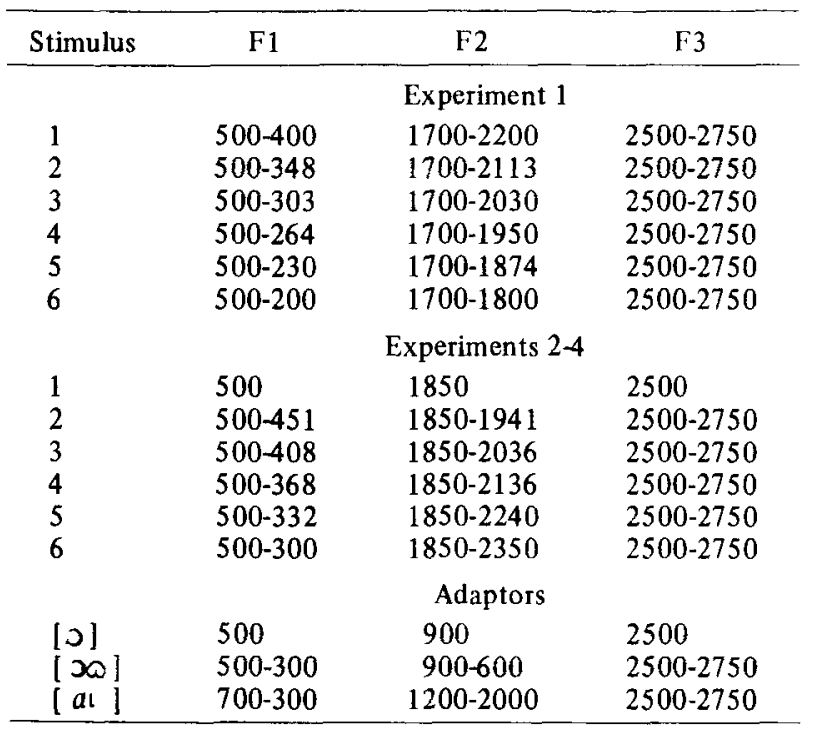

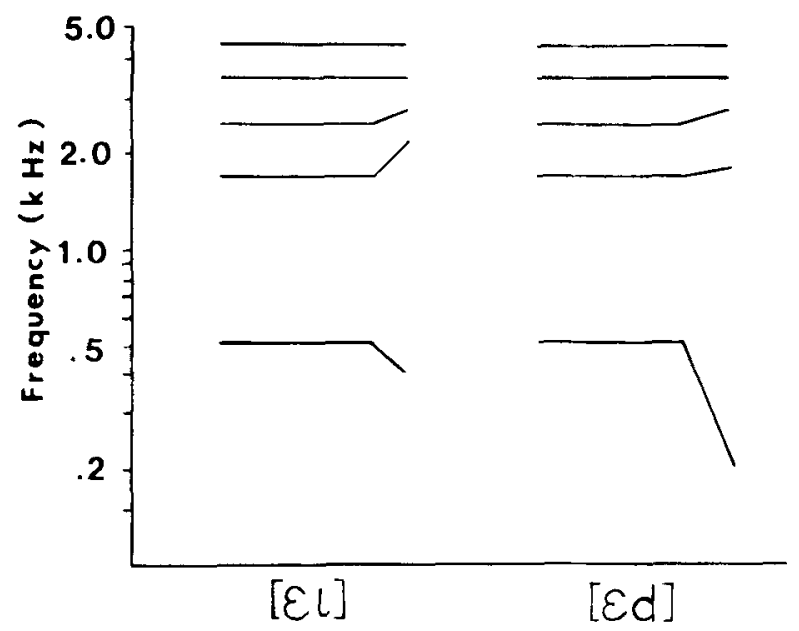

Figure 1. Schematic spectrograms of the endpoint stimuli (numbers 1 and 6) of the $|\varepsilon t-\varepsilon d|$ series of Experiment 1. These were also the adaptors.

tests were also constructed, one with Stimulus 1 as adaptor and one with Stimulus 6. Adapting stimuli were repeated 200 times at the beginning of the test and 100 times between blocks of test stimuli thereafter, with a $400-\mathrm{msec}$ ISI; test stimuli were presented six at a time, in 10 blocks, for a total of 60 adapted identification responses per test.

Tapes were played on a Sony TC-353D stereo tape deck; individual subjects listened binaurally over TDH-49 headphones at $80 \mathrm{~dB}$ SPL, measured for the steady-state vowel. They marked a preprinted answer sheet containing letter symbols and key words for the sounds. After the test procedure was explained, each subject listened to several practice items; all reported that the stimuli were acceptable as tokens of the sounds indicated.

Each subject took one baseline and one adaptation test in a single 30-min session and the second baseline and adaptation tests in a session at least a day later. Order to adaptors was counter balanced across subjects.

\section{Results}

Both Stimulus 1, perceived as the diphthong $[\varepsilon l]$, and Stimulus 6, perceived as the vowel plus consonant
(VC) syllable $[\varepsilon d]$, produced comparable adaptation effects. As can be observed in Figure 2, the identification boundary was shifted in each case toward the adaptor category, and the effects are quite symmetrical. Thus, for example, by the majority of judgments in both baseline conditions, Stimulus 3 was called a vowel and Stimulus 4 a vowel plus consonant, while under adaptation each of those judgments was reversed once: Stimulus 3 was called $[\varepsilon d]$ when $[\varepsilon \iota]$ was the adaptor, and Stimulus 4 was identified as $[\varepsilon l]$ when $[\varepsilon d]$ was the adaptor.

The data from each session were subjected to a two-way analysis of variance, which showed the difference between baseline and adapted conditions to be significant in both cases [for the [ $\varepsilon$ ] adaptor, $F(1,11)=12.14, p<.005$; for the $[\varepsilon d]$ adaptor, $F(1,11)$ $=133.65, p<.0005]$. In addition, the crossover points, or category boundaries, were estimated by linear interpolation for each subject in each session. These values were used to compute boundary shifts and to perform matched-pairs $t$ tests for each adaptor condition. Adaptation with Stimulus 1, the diphthong $[\varepsilon t]$, shifted the identification boundary by .91 stimulus units on the abscissa, which was statistically significant $[\mathrm{t}(11)=3.284, \mathrm{p}<.01$, two-tailed]. The shift induced by adaptation with Stimulus 6, the VC syllable $[\varepsilon \mathrm{d}]$, was 1.12 stimulus units, also significant $[\mathrm{t}(11)=5.206, \mathrm{p}<.001]$.

\section{Discussion}

The fact that a stimulus perceived as a vowel and another perceived as a VC syllable have approximately equal and opposite effects as adaptors on the series of sounds between them would certainly argue strongly against an interpretation of adaptation shifts in terms of linguistic feature detectors with phonetic segments as their domain. The listeners' judgments appear, instead, to acknowledge a continuum of "degrees of closure" between the articulatory gesture for a close vowel and the total closure for a stop, at least in syllable-closing position, rather than an absolute dichotomy between consonants and vowels.
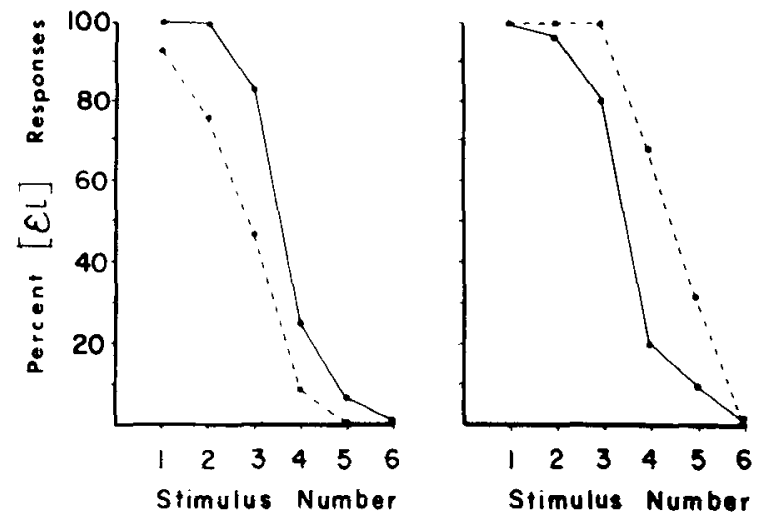

Figure 2. Identification functions from Experiment 1: control (solid) and adapted (dashed) functions with adaptation by Stimulus 1 (left) and Stimulus 6 (right). 
The terminal frequency of the F1 transition is the acoustic cue which most directly reflects degree of closure. Thus, after repeated listening to Stimulus 6, with a very low terminal frequency of $F 1$, listeners were inclined to hear intermediate values as ending in a close vowel rather than a voiced stop-as if their criterion for how low F1 must be to signify "closure" had become more strict. Likewise, exposure to Stimulus 1 , with its shallower F1 transition, caused listeners to adjust their criterion in the opposite direction, accepting more stimuli as examples of stop closure than before.

Such an interpretation, based on analog values of particular acoustic parameters, appears compatible with a purely auditory explanation of adaptation boundary shifts, as suggested by Simon and StuddertKennedy (1978), but it raises certain questions as well. For example, the stimuli of Experiment 1, though varying in phonological structure from $\mathrm{V}$ to $\mathrm{VC}$, all terminated in a brief $(35-\mathrm{msec})$ episode of spectral change (formant transitions). Is the presence of some such (typically consonantal) cue essential to obtain adaptation effects? If auditory short-term memory constraints are, indeed, a principal factor in adaptation (Sawusch \& Nusbaum, 1979), then we might expect sounds containing brief transitions to behave similarly, while sounds without such cues (e.g., steady-state vowels) might show a qualitative difference.

\section{EXPERIMENT 2}

This experiment was designed to compare the effects of two adaptors, the extremes of a continuum from a steady-state $[\varepsilon]$ to an $[\varepsilon l]$, on the sounds of the continuum. This reversed the conditions of the previous experiment, in that all stimuli had the same phonemic status (i.e., vowel), but the acoustic cue went from unchanging to changing formant transitions, the amount of frequency change being the only variable parameter.

\section{Method}

Stimuli. A six-place continuum from $[\varepsilon]$ to $[\varepsilon t]$ was synthesized. All stimuli were $81 \mathrm{msec}$ long. Stimulus 1 , a steady-state vowel, had formant frequencies of $500 \mathrm{~Hz}(\mathrm{~F} 1), 1,850 \mathrm{~Hz}(\mathrm{~F} 2)$, and $2,500 \mathrm{~Hz}(\mathrm{~F} 3)$. The other five stimuli had these same formant frequencies for $46 \mathrm{msec}$, followed by 35 -msec transitions in which $F 1$ fell and $F 2$ and $F 3$ rose. The amount of frequency transition for $F 1$ and F2 increased in equal logarithmic steps through Stimulus 6, which had transitions of 500 to $300 \mathrm{~Hz}(\mathrm{Fl})$ and $1,850-2,350 \mathrm{~Hz}(\mathrm{~F} 2)$; $F 3$ transitions were $2,500-2,750 \mathrm{~Hz}$ for Stimuli 2 through 6 . Fourth and fifth formant frequencies_were fixed at 3,500 and $4,500 \mathrm{~Hz}$, and pitch rose linearly from 105 to $118 \mathrm{~Hz}$. See Table 1 for values and Figure 3 for schematic spectrograms of the endpoint stimuli.

Subjects. Twelve persons from the same population as Experiment 1 served as subjects, including two from the first group.

Procedure. The procedure and apparatus were essentially identical to those in Experiment 1.

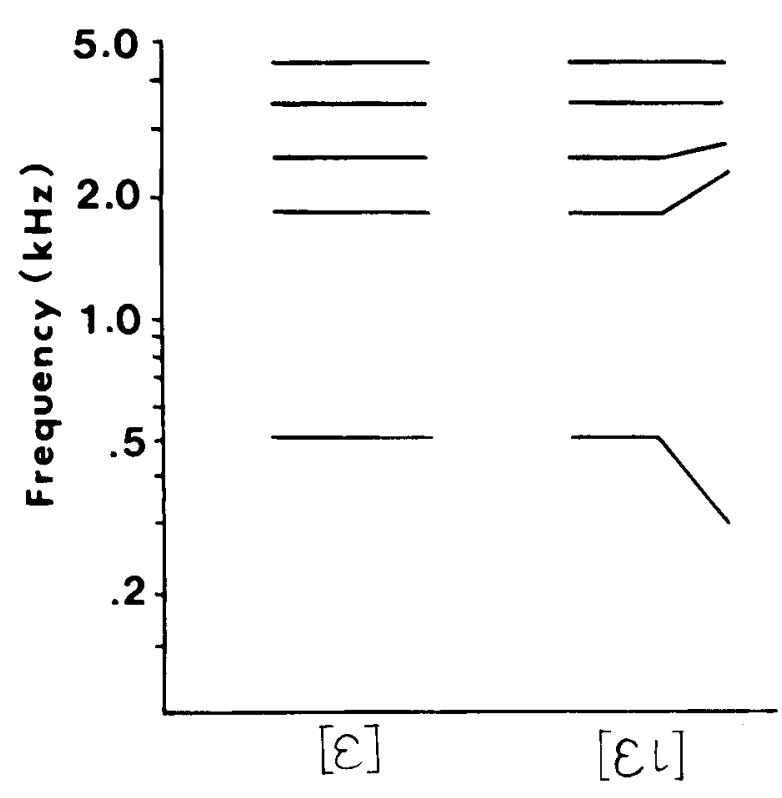

Figure 3. Schematic spectrograms of the endpoint stimuli of the $[\varepsilon-\varepsilon 1]$ series of Experiments 2 and 4 . These were also the adaptors in Experiment 2.
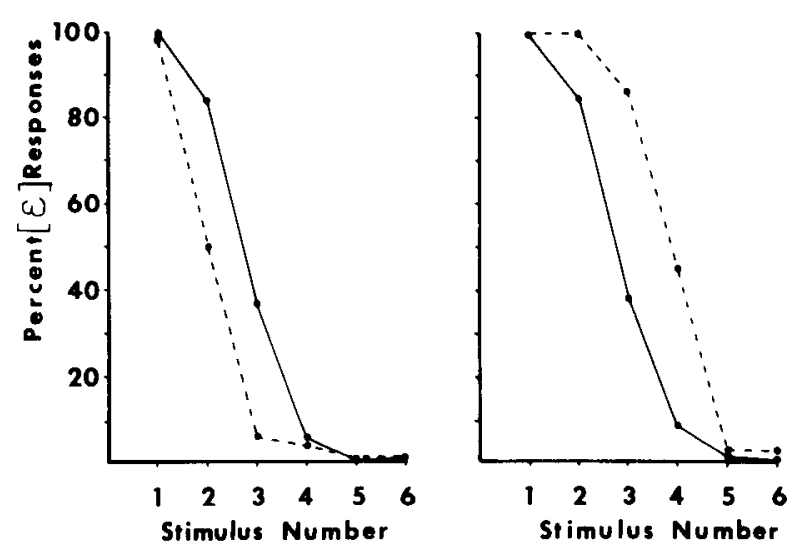

Figure 4. Identification functions from Experiment 2: control (solid) and adapted (dashed) functions with adaptation by Stimulus 1 (left) and Stimulus 6 (right).

\section{Results}

As can be seen in Figure 4, both Stimulus 1, the short steady-state $[\varepsilon]$, and Stimulus 6 , the short diphthong $[\varepsilon l]$, proved effective as adaptors. Analyses of variance showed both differences between baseline and adapted scores to be highly significant [for adaptation by Stimulus $1, F(1,11)=40.76, p<.005$; for Stimulus 6, $F(1,11)=35.75, p<.0005$ ]. The estimated crossover values showed a boundary shift of .71 stimulus unit after adaptation by Stimulus 1, a significant change in the predicted direction $[\mathrm{t}(11)=$ $3.729, \mathrm{p}<.01$ ]; the shift in the opposite direction after adaptation by Stimulus 6 was 1.01 stimulus units, also a significant change $[\mathrm{t}(11)=6.533$, $\mathrm{p}<.0011$. 


\section{Discussion}

Clearly, then, judgments of vowel identity along a continuum of dynamic rather than quality differences are subject to selective adaptation by the endpoint sounds, at least for stimuli of very short duration. Repeated exposure to the steady-state vowel appears to affect listeners' judgments of how much dynamic change is sufficient to cue a diphthong, and exposure to vowels ending in greater frequency transitions affects those judgments conversely. It is therefore not necessary for a speech sound to contain any particular typically consonantal acoustic attribute in order to be an effective adaptor of other sounds. This is true even when the adapted sounds do contain such an acoustic property. There might, of course, be differences in the relative effectiveness of adaptors with and without such acoustic properties. Unfortunately, the data of Experiment 2 cannot illuminate this question, since the asymmetry of the boundary shifts could well be due to a "floor effect" caused by the range and step sizes of the stimulus values chosen.

The data of Experiments 1 and 2 taken together suggest that there is no difference, in principle, between adaptation-induced boundary shifts for consonants and for vowels; sounds from each class can affect members of both classes in the same manner, and apparently to a similar degree, under a given set of experimental conditions. In general, the data are adequately explained in auditory terms alone; if phonetic classification play any role, it must be a minor one. But perhaps we can gain some further insight into what is being contrasted-that is, what form of signal representation serves as the locus of the change in criterion-by attempting cross-series adaptation with these stimuli. In the following experiments, the $[\varepsilon-\varepsilon l]$ continuum was used to test the effectiveness of four adaptors which resembled the endpoint stimuli in specific ways.

\section{EXPERIMENT 3}

\section{Method}

Stimuli. The test series, a six-point continuum from $[\varepsilon]$ to $[\varepsilon i]$, was similar to the series of Experiment 2, except that the total duration was longer $(140 \mathrm{msec})$, while the final transitions remained brief $(40 \mathrm{msec}) ; \mathrm{f}_{0}$ fell linearly from 118 to $100 \mathrm{~Hz}$. The adaptors were a steady-state [o] vowel, identical to Stimulus 1 of the test series except for the frequency of F2, which was $900 \mathrm{~Hz}$, and a diphthong [oo], identical to Stimulus 6 except for the frequency of F2, which was $900 \mathrm{~Hz}$ for the steady-state portion and fell to $600 \mathrm{~Hz}$ over the last $40 \mathrm{msec}$. See Table I for values and Figure 5 for schematic spectrograms of the adaptors.

Subjects. Twelve volunteers from the same population served as subjects. Four had participated in a previous experiment.

Procedure. Essentially the same procedure and apparatus were employed as in the previous experiments.

\section{Results}

As Figure 6 shows, neither the steady-state [?] nor the diphthong [DQ] was effective as an adaptor of the

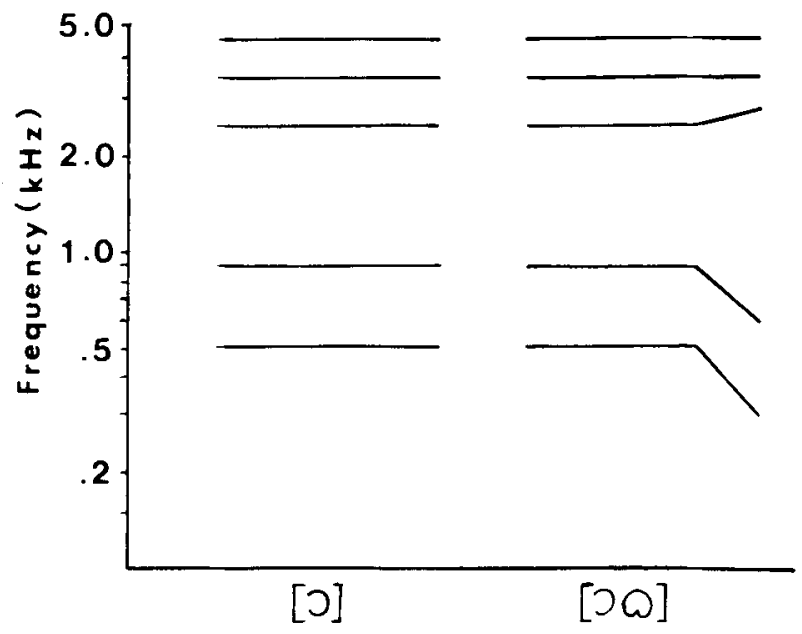

Figure 5. Schematic spectrograms of the adaplors $[\supset \mid$ and [xa] of Experiment 3.
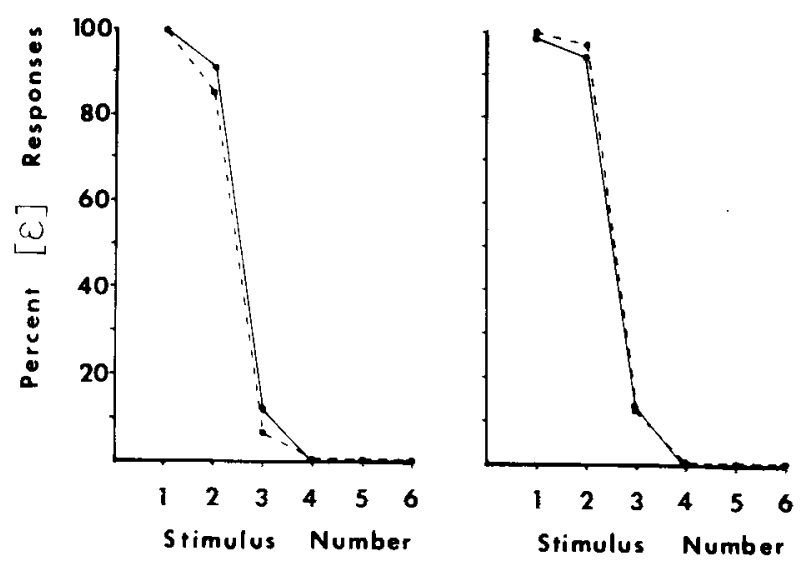

Figure 6. Identification functions from Experiment 3: control (solid) and adapted (dashed) functions with adaptation by [o] (left) and (כo) (right).

$\left[\varepsilon-\varepsilon_{1}\right]$ continuum. Analyses of variance confirmed that the baseline and adapted conditions were not significantly different for either adaptor [for [ $\supset$ ], $F(1,11)=1.63, p>.10$; for $[\infty], F(1,11)=.34, p>$ .10]. The boundary values differed by only slight and insignificant amounts from the control values: .10 stimulus unit for $[0][t(11)=1.018, p>.10]$ and .01 stimulus unit for $[20][t(11)=.308, p>.10]$.

\section{Discussion}

The failure of the two adaptors in Experiment 3 to cause a cross-series shift in identification might be explained in several ways. One would be that crossseries effects depend on shared consonantal "features" and cannot occur with vowels, or that the adaptors did not share enough phonological features with the test sounds. Likewise, insufficient acoustic similarity might be responsible; although first formant frequencies were identical, the second formants differed by an octave and, in the case of $[\mathrm{p} \sigma]$, contained a transition in the direction opposite from that of the 
adapted series. Finally, the duration difference might explain the lack of an effect: Perhaps the 140-msec stimuli were more stable, and hence less adaptable, than the $81-\mathrm{msec}$ versions due to the relative qualities of their representation in short-term memory. The adaptors of Experiment 4 were designed to test these alternative explanations.

\section{EXPERIMENT 4}

In this experiment, cross-series adaptation was attempted with two more adaptors, both designed to be similar to Stimulus 6 of the test series; any adaptation shift, therefore, would be predicted to be toward a higher stimulus number. Both the test and the adaptors were of short duration $(81 \mathrm{msec})$, as in Experiment 2; one adaptor was simply a short [ $\mathrm{OC}$, and the second was an equally brief $[a \imath]$.

\section{Method}

Stimuli. The test series had exactly the same acoustic characteristics as the stimuli of Experiment 2, brief vocalic sounds varying from steady-state $[\varepsilon]$ to $[\varepsilon t]$. The first adaptor had the same steady-state and transitional frequencies as the [O०] of Experiment 3, but the same duration and fundamental frequency as the test stimuli. Likewise, the second adaptor also was $81 \mathrm{msec}$ long (of which $46 \mathrm{msec}$ was steady state and $35 \mathrm{msec}$ transitions) and also had $f_{0}$ rising linearly from 105 to $118 \mathrm{~Hz}$. The steadystate formants for the initial portion of the $\left[a_{t}\right]$ adaptor were $700 \mathrm{~Hz}(\mathrm{~F} 1), 1,200 \mathrm{~Hz}(\mathrm{~F} 2)$, and $2,500 \mathrm{~Hz}(\mathrm{~F} 3)$; they ended with linear transitions terminating at $300,2,000$, and $2,750 \mathrm{~Hz}$, respectively. See Table 1 for further details.

Subjects. Twelve subjects from the same population took the two sets of tests. Three had participated in one of the previous tests.

Procedure. Procedure, including counterbalanced order of adaptors, and apparatus were the same as described previously.

\section{Results}

Shortening the $[\mathrm{a} a]$ adaptor did not result in a crossseries adaptation effect, as can be seen in the left panel of Figure 7. The overall change in responses was slight and not statistically significant; the boundary shift was .06 stimulus units in the direction opposite that predicted and also was not significant.
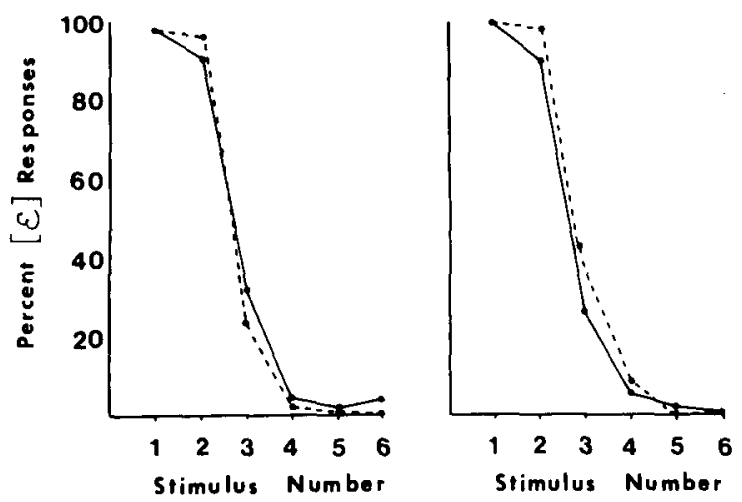

Figure 7. Identification functions from Experiment 4: control (solid) and adapted (dashed) functions with adaptation by $[\supset \infty]$ (left) and [al] (right).
The $\left[a_{t}\right]$ adaptor, on the other hand, did produce a significant change in responses in the predicted direction, as measured by analysis of variance $[\mathrm{F}(1,11)=$ $4.89, \mathrm{p}<.05]$. The boundary shift of .31 stimulus unit, though only about one-third of that obtained with the endpoint $[\varepsilon l]$ in Experiment 2, was also significant $[\mathrm{t}(11)=2.251, \mathrm{p}<.05]$.

\section{Discussion}

The fact that cross-series adaptation effects, which have previously been found only for consonants, can occur with vowels as well, suggests a common origin for the effect. It would appear that the adaptor $\left[a_{l}\right]$ was perceived as sufficiently similar to the $[\varepsilon l]$ stimuli of the $[\varepsilon-\varepsilon l]$ test series that the subjects' criterion for identifying a stimulus as $[\varepsilon t]$ was made stricter by exposure to the $\left[a_{1}\right]$. But the same amount of exposure to the [Do] adaptor did not have this effect, which makes the results all the more interesting.

It is difficult to see how the effectiveness of the [ai] adaptor could be due to "peripheral fatigue," given its spectral dissimilarity from the test syllables for most of its duration. Only in the terminal formant transitions do the $[a t]$ and $[\varepsilon l]$ stimuli contain energy at similar frequencies. It is true that several studies suggest that such transitions create an auditory percept dominated by their terminal frequency (Stevens, 1975). Brady, House, and Stevens (1961) showed, for example, that if subjects are required to match the pitch of a tone to rising or falling transitions, their estimates are biased toward the final frequency. A similar result was found under similar conditions with tone glides by Nábělek, Nábělek, and Hirsh (1970). Since the final transitions of $\left[a_{1}\right]$ and $\left[\varepsilon_{1}\right]$ are in the same directions (F1 falling, F2 and $F 3$ rising) and terminate in similar frequencies, it might be argued that this constitutes sufficient spectral overlap to generate peripheral fatigue. It should be possible to explore this experimentally, using slower transitions and various ratios of duration between steady and changing portions. But first we should entertain the possibility that the similarity is at a more abstract level, although still below the level of linguistic features. For example, if the pattern of diverging $F 1$ and $F 2$ forms a unitary auditory percept or pattern, independent of the frequencies involved, as opposed to a pattern of falling $F 1$ and F2, this could serve as the basis for the cross-series effect found with $\left[a_{1}\right]$ but not with $[00]$. This is the kind of solution suggested, for example, by Bryant (1978), who concludes that holistic similarity of patterns, rather than analytic feature-by-feature comparison, better explains the adaptive effects in his experiments. Sawusch (1977) also interprets the effects of his "spectrally displaced" consonantal adaptors in this way. Perhaps the most direct test of this explanation would be to adopt the methodology of Sawusch (1977, Experiment 2) and present the $[a t]$ and endpoint $[\varepsilon t]$ 
adaptors to the same and to opposite ears from the test sounds. If the abstract pattern of diverging formants is the source of the effect with $\left[a_{1}\right]$, we should see $100 \%$ interaural transfer due to central auditory contrast with the $\left[a_{l}\right]$ adaptor, but not with $\left[\varepsilon_{1}\right]$, which would also cause fatigue.

The failure of the [20] adaptor to affect identification is somewhat more difficult to explain. If more evidence were needed against a linguistic feature detector interpretation, we might dwell on the traditional phonemic description of $[\varepsilon l]$ and $[\partial \omega]$ as monophonematic $/ \mathrm{e} /$ and $/ \mathrm{o} /$, differing only by the feature "front" vs. "back," as opposed to /ai/ as a sequence of two phonemes. On the level of auditory contrast, we have suggested that the abstract $F 1-F 2$ patterns (diverging vs. falling) are a likely basis for the effectiveness of $\left[a_{1}\right]$ in contrast to [DQ] in adapting the $[\varepsilon-\varepsilon 1]$ series. However, with the [Jol adaptor, an effect due to peripheral fatigue might have been expected on the basis of substantial spectral overlap with the test sounds. For comparison, Figure 8 shows schematic spectrograms of Stimulus 6 flanked by the two adaptors $\left[a_{l}\right]$ and $[20]$. The $\left.p o\right]$ adaptor and Stimulus 6 have identical F1 frequencies throughout their duration. (The third formant was identical in all stimuli, with a $2,500-\mathrm{Hz}$ steady-state portion followed by a transition to $2,750 \mathrm{~Hz}$. This was done simply for technical neatness; a rising F3 is not natural in [30], but was not an effective cue in any case, since F3 was more than $35 \mathrm{~dB}$ less intense than F1.)

The lack of an effect with the [OO] adaptor would appear to be a sign, albeit a negative one, of great frequency specificity in the fatigue effect, demanding more acoustic similarity between adaptor and test stimuli than was present in Experiment 4. If so, this would appear to constitute a difference from results with consonantal series, in which, for example, adaptors consisting of only the higher formants (with F1 missing) cause shifts in consonant identification (see Ades, 1976, for a review). If we view the $[\varepsilon-\varepsilon t]$ series

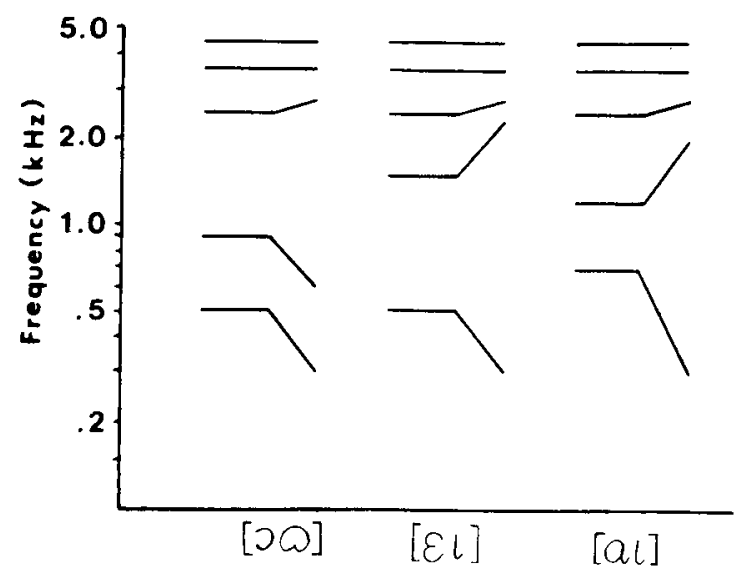

Figure 8. Schematic spectrograms of Stimulus 6 of Experiment 4 (center), flanked by the adaptors $|>\infty|$ (left) and $|a|$ (right). as a continuum of "no change" vs. "change," the frequency transitions of F1 alone would suffice to cue this distinction. Thus, the F1 transitions in [Da], given their complete spectral overlap in the Fl region, ought to have a fatiguing effect, regardless of the dissimilarities in $\mathrm{F} 2$.

Grounds for an explanation, however, may be found in the results of an experiment by Bailey (1973) with two sets of [ba-da] consonant stimuli. In one series ("Fixed F2"), the place of articulation was cued by varying $F 3$ transitions, $F 2$ being kept fixed, while in the second series ("No F3") place was cued by F2 transitions and no formant was present in the spectral region of F3. Bailey found that endpoint stimuli from the No F3 series adapted responses to the Fixed F2 series but that those from the Fixed F2 series, when used as adaptors, had almost no effect on the perceived identity of the No F3 series. (A small d shift was attributed to the effect of the slightly falling fixed F2 transition, not to F3.). This asymmetry indicates that when the adaptor contains energy in a given spectral region and the test stimuli do not, the perception of the test stimulus is not altered in that respect. Thus, the F2 of [Do] should not affect the perception of the $\left[\varepsilon-\varepsilon_{l}\right]$ stimuli, but $F 1$ should. However, this would leave the F2 of the test stimuli unaffected, and, for the vowel vs. diphthong distinction, the absence or presence of a transition in F2 is by itself a completely sufficient cue. Subjects thus presumably heard the test sounds of the $[\varepsilon-\varepsilon l]$ series with fatigued peripheral receptors in the $F 1$ region and unaffected receptors in the $F 2$ region $(1,850-2,350 \mathrm{~Hz})$ and responded $[\varepsilon l]$ whenever they detected a frequency transition sufficient to cue a diphthong.

\section{GENERAL DISCUSSION}

The results of Experiments 1 and 2 demonstrate that adaptation effects, such as have been observed with consonantal sounds in CV and VC syllables and with isolated steady-state vowels, can be induced also in series of synthesized speech sounds varying from diphthong to vowel plus consonant or from steadystate vowel to diphthong. This result fits with a great deal of other accumulated evidence that the linguistic feature composition of a speech stimulus is not directly involved in producing the change in responses-an auditory level of representation is more likely.

Remez (1979), for example, has shown that even a continuum in which formant bandwidths are widened in steps to create a series from a vowel to a nonspeech "buzz" is equally susceptible to adaptation by both endpoint sounds. Identical results were found for a series varying from [ba] to nonspeech "buzz" in Remez (1980), providing further evidence that, even at the auditory level, any use of the term "feature" 
to describe the basis of adaptive effect with speech sounds must be so qualified that it can hardly have any straightforward phonetic interpretation.

The simplest explanation of the results of the first two experiments, and one which fits readily into the frame of reference proposed by Simon and StuddertKennedy (1978), is that the perceived amount of frequency change in the formants (principally $F 1$ in Experiment 1, F1 and F2 in Experiment 2) is made to seem greater or less by contrast with the amount of change in the repeated adaptor. Since these adaptors were drawn from the test series, they provide no grounds for distinguishing between "central auditory contrast" and "peripheral fatigue" as the origin of the effects observed: by analogy with consonantal adaptation, one might expect both to be involved.

In Experiments 3 and 4, adaptors were employed which differed from the test sounds in specific ways. The results, though not entirely conclusive, are at least compatible with the view that adaptation effects with these vocalic stimuli have the same dual origin as with consonantal sounds. The small, but significant, effect of the $\left[a_{1}\right]$, with its diverging pattern of F1-F2 transitions and lack of spectral overlap with the test sounds, can be likened to the effect of the spectrally displaced consonantal adaptors of Sawusch (1977), that is, central contrast between abstract auditory patterns. In the case of [0] and [Do], in which the abstract patterns differ but partial spectral overlap exists, negative results were found. Interpreting the lack of an effect is, of course, hazardous, but there is no compelling reason to expect differences in the response of peripheral receptors to vocalic as opposed to consonantal stimuli. Thus, given the findings of Bailey (1973) with consonants, that the acoustic cues contained in a particular formant are immune to adaptive fatigue when the adaptor contains no energy in that formant's spectral vicinity, we may conclude that any peripheral fatigue that took place in the $F 1$ region was rendered ineffective by the unmodified perception of the F2 cue. Whether or not the effect of such "selective peripheral fatigue" would be observable if rating responses were used (as in Sawusch, 1977; Sawusch \& Nusbaum, 1979) is a question that should be addressed in future experiments.

If adaptation effects on consonantal and vocalic sounds have the same origin and are at least partially identical to anchoring effects (as Simon \& StuddertKennedy, 1978, suggest), further experiments are also needed to more fully exploit this type of stimulus, in which both steady-state spectral cues and formant frequency changes jointly contribute to phonetic identity. Informal tests suggest that acceptable diphthongs can be synthesized with a considerable range of variation in the durations of the steadystate and varying portions. Thus, phonetically identical stimuli making different demands on auditory memory could be compared and used to test Sawusch and Nusbaum's (1979) contention that "the processes underlying the anchoring effect found with vowels may be quite different from the adaptation effects found with consonants (p. 301). The data of this study, while not conclusive on this point, suggest that the difference may be one of degree rather than kind. But Sawusch and Nusbaum, in the same article, raise the issue which is probably most crucial to resolving this question. Speculating on the applicability of Restle's (1978) model of adaptation level theory, they note that it incorporates "the influence of both information from immediately preceding stimuli (auditory memory) and prototypes in long-term memory into one unified auditory ground against which new stimuli are compared"'(p. 310). Now, while specific acoustic details of adaptor and test stimuli are often critical in determining whether adaptive changes occur, the long-term prototype must be stored in rather abstract form in order to be useful. We may well ask how specific stimuli (adaptors or anchors) could directly modify a template or prototype in long-term memory, whether some form of buffered image evoked from, or based on, the prototype, but capable of shortto medium-term modification (such as adapting to speaker characteristics or dialect), must mediate the process, serving as the locus of auditory contrast. Such an image would, indeed, correspond to the factor B in Restle's (1978) model, as Sawusch and Nusbaum suggest. We are far from being able to test this model quantitatively with speech; the effects of such variables as phonetic acceptability of the stimuli and instructional set (Sawusch \& Pisoni, 1976) and others (corresponding to Restle's factor $\mathrm{K}$ ) would have to be known and fixed for a given experiment before one could begin to test the model with speech sounds. But the power of the model, demonstrated by Restle in accounting for both assimilative and contrastive effects in experiments with visual sizecontrast illusions, suggests that it might subsume the "auditory contrast" explanation of speech adaptation results advanced by Simon and Studdert-Kennedy (1978) and others. Once the quantitative limits are known, selective adaptation might prove most valuable as an objective method of measuring perceived auditory differences among speech cues or the relative strengths of various cues to the same "feature" of a prototype in long-term memory.

\section{REFERENCE NOTE}

1. Pisoni, D. B. On the nature of categorical perception of speech sounds (Supplement to Status Report on Speech Research). New Haven, Conn: Haskins Laboratories, 1971.

\section{REFERENCES}

Ades, A. E. Adapting the property detectors for speech perception. In R. J. Wales \& E. Walker (Eds.), New approaches to language mechanisms. Amsterdam: North-Holland, 1976. 
BAiley, P. J. Perceptual adaptation for acoustical features in speech. Speech Perception (Department of Psychology, Queen's University, Belfast), 1973, 2, 29-34.

Bailey, P. J. Perceptual adaptation in speech: Some properties of detectors for acoustical cues to phonetic distinctions. Unpublished PhD dissertation, University of Cambridge, 1975.

Brady, P. T., House, A. S., \& Stevens, K. N. Perception of sounds characterized by a rapidly changing resonant frequency. Journal of the Acoustical Society of America, 1961, 33, $1357-1362$.

Bryant, J. S. The feature detection process in speech perception. Journal of Experimental Psychology: Human Perception and Performance, 1978, 4, 610-620.

Darwin, C. J., \& Baddeley, A. D. Acoustic memory and the perception of speech. Cognitive Psychology, 1974, 6, 41-60.

Diehl, R. L., Elman, J. L., \& McCusker, S. B. Contrast effects in stop consonant identification. Journal of Experimental Psychology: Human Perception and Performance, 1978. 4, 599-604.

Fry, D., Abramson, A., Eimas, P. D., \& Liberman, A. L. The identification and discrimination of synthetic vowels. Language and Speech, 1962, 5, 171-189.

Godfrey, J. J. Perceptual difficulty and the right ear advantage for vowels. Brain and Language, 1974, 4, 323-336.

LAdEFoged, P., \& Broadbent, D. E. Information conveyed by vowels. Journal of the Acoustical Society of America, 1957, 29, 98-104.

Morse, P. A., Kass, J. E., \& Turkienicz, R. Selective adaptation of vowels. Perception \& Psychophysics, 1976, 19, 137-143.

NábĚleK, I., NábĚleK, A., \& HirSh, J. J. Pitch of tone bursts of changing frequency. Journal of the Acoustical Society of America, 1970, 48, 536-553.

Pisoni, D. B. Auditory and phonetic memory codes in the discrimination of consonants and vowels. Perception \& Psychophysics, 1973, 13, 253-260.

RABINER, L. R. Digital formant synthesizer for speech synthesis studies. Journal of the Acoustical Society of America, 1968, 43, 822-828.
Remez, R. E. Adaptation of the category boundary between speech and nonspeech: A case against feature detectors. Cognitive Psychology, 1979, 11, 38-57.

REMEZ, R. E. Susceptibility of a stop consonant to adaptation on a speech-nonspeech continuum: Further evidence against feature detectors in speech perception. Perception \& Psychophysics, $1980,27,17-23$.

RestLe, F. Assimilation predicted by adaptation-level theory with variable weights. In N. J. Castellan \& F. Restle (Eds.), Cognitive theory (Voi. 3). Hillsdale, N.J: Erlbaum, 1978.

Sawusch, J. R. Peripheral and central processes in selective adaptation of place of articulation in stop consonants. Journal of the Acoustical Society of America, 1977, 62, 738-750.

Sawusch, J. R., \& Nusbaum, H. C. Contextual effects in vowel perception 1: Anchor-induced contrast effects. Perception \& Psychophysics, 1979, 25, 292-302.

Sawusch, J. R., \& Pisoni, D. B. Response organization and selective adaptation to speech sounds. Perception \& Psychophysics, 1976, 20, 413-418.

Simon, H. J., \& Studdert-Kennedy, M. Selective anchoring and adaptation of phonetic and nonphonetic continua. Journal of the Acoustical Society of America, 1978, 64, 1338-1357.

Stevens, K. N. The potential role of property detectors in the perception of consonants. In G. Fant \& M. A. A. Tatham (Eds.), Auditory analysis and perception of speech. New York: Academic Press, 1975.

Weiss, M. S., \& House, A. S. Perception of dichotically presented vowels. Journal of the Acoustical Society of America, $1973,53,51-58$.

Williams, P. D., \& Sharf, D. J. Effect of adaptation on the perception and production of vowel duration preceding stop consonants. Journal of Phonetics, 1979, 7, 81-92.

(Received for publication January 8, 1980; revision accepted May 21, 1980.) 